\title{
Evaluation of Phenotypic Methods to Identify Extended Spectrum Beta-lactamase (ESBL) Producing Gram negative Bacteria
}

\author{
Shikha Paul ${ }^{1}$, Sanya Tahmina Jhora ${ }^{1}$, Prashanta Prasun Dey ${ }^{2}$ \\ ${ }^{1}$ Department of Microbiology, Sir Salimullah Medical College, Dhaka \\ ${ }^{2}$ Department of Endocrinology, Kumudini Hospital, Tangail
}

\begin{abstract}
Extended spectrum beta-lactamase (ESBL) producing Gram negative bacteria are usually multiple drug resistant and their cephalosporin and aztreonam resistance is not reliably detected by susceptibility tests.This study was carried out to find out a cost effective easy standard method to identify ESBL producing bacteria in a laboratory associated with tertiary care hospital and to determine the incidence of ESBL positive bacteria isolated from different clinical specimens. Thereby isolated 124 Gram negative bacteria from various samples were subjected to screening test, double disc synergy test (DDST)and E test ESBL method.Screening test detected $69.35 \%$, DDST identified $37.1 \%$ and $E$ test ESBL method confirmed $55.65 \%$ ESBL producing strains. Screening test and DDST was compared to $\mathbf{E}$ test ESBL method. Considering $\mathrm{E}$ test as standard the sensitivity and specificity of screening test were $97.10 \%$ and $65.45 \%$ respectively and that of DDST were $\mathbf{6 2 . 3 1 \%}$ and $\mathbf{9 4 . 5 5 \%}$ respectively. Low specificity of screening test reflects detection of many false positive strains and low sensitivity of DDST signals many missed identification. This study suggested the use of $E$ test ESBL method to confirm screening positive ESBL isolates at microbiology laboratory.
\end{abstract}

Key words: ESBL, DDST, Etest.

\section{Introduction:}

Beta-lactamases are the primary cause of bacterial resistance to beta-lactam antibiotics ${ }^{1}$. These are heterogeneous group of resistance enzymes. Till now more than 890 distinct betalactamases have been identified ${ }^{2}$.

Resistance to beta-lactam antibiotics is prevalent among many Gram negative bacteria mostly because they inherently produce beta-lactamases enzymes which hydrolyze the betalactam ring and inactivate antibiotics ${ }^{1}$. Many new beta-lactam antibiotics have been developed that are specifically designed to be resistant to the hydrolytic action of beta-lactamases. With development of each new class to treat patients, new beta-lactamases are emerged which are resistant to that class of new drug ${ }^{3}$.

\section{Correspondence:}

Dr. Shikha Paul

Associate Professor

Department of Microbiology, SSMC, Dhaka

Email:shikhapaul2013@gmail.com

Phone:01732806772
Increasing incidence of extended spectrum beta lactamase (ESBL) producing strains is being reported all over the world. Higher incidences are reported in intensive care units, renal failure, burn, urinary catheter ${ }^{3}$. Several studies of Bangladesh reported significant number ESBL producing strains among gram negative bacilli ${ }^{4,5}$. Identification of ESBL producing Gram negative bacteria provides valuable information to the clinicians and raises demand for contact precautions to avoid hospital transmission ${ }^{6}$. ESBL producers can be falsely susceptible to identification discs in routine tests making its recognition difficult ${ }^{7}$.

ESBLs can be detected by phenotypic and genotypic methods. Routine identification at clinical laboratory depends on phenotypic methods. The recommended phenotypic strategy for ESBL detection includes initial screening followed by confirmation. Screen tests are Disk diffusion and Broth microdilution. Disk diffusion utilizes cefotaxime or cefodoxime or ceftadizime or aztreonam disk. Use of more than one antimicrobial disk for screening improves the sensitivity of ESBL detection. Phenotypic confirmation can also be done by Disk diffusion and Broth microdilution. 
Confirmatory testing requires the use of either cefotaxime or ceftadizime alone and in combination with clavulanic acid. These tests are standardized for Escherichia coli, Klebsiella pneumonia, Klebsiella oxytoca and Proteus mirabilis. Members of Enterobacteriaceae with inducible AmpC lactamase producing capability need cefepime alone and in combination with beta lactamase inhibitor to confirm as ESBL producer ${ }^{8}$. Other confirmation methods are combined disk test(CDT), Double disk synergy test(DDST), ESBL gradient test, E test for ESBL, Three dimensional test etc ${ }^{3,9}$.

This study employed screening test, double disc synergy test (DDST) and E test ESBL methods to identify ESBLs producing isolates. E test was taken as standard and screening test and DDST were compared to E test ESBL method to evaluate them against $\mathrm{E}$ test ESBL method.

\section{Methods:}

Total 124 Gram negative bacteria were isolated from different biological samples such as urine, pus, wound swab, stool, blood and High vaginal swab (HVS)of patients attending microbiology laboratory of Sir Salimullah Medical College(SSMC), Dhaka during the period of March 2013 to August 2013.Cultures of these samples were advised by physicians ofinpatient and outpatient departments of SSMC \& Mitford Hospital.Standard methods were employed for collection of samples, isolation and identification of the organisms. MaConkey's agar and blood agar media were used for primary isolation. Triple sugar iron (TSI), Motility indole urea(MIU), Oxidase, Gram stain from colony wereused for identification of Gram negative bacilli. Finally extended spectrum beta lactamase (ESBL) producing strains were recognized by Screening test, ${ }^{2,7}$ Double disc synergy test $(\mathrm{DDST})^{2,7,10}$ and E test ESBL method ${ }^{3,11,12}$.

\section{Reference strains:}

Klebsiella pneumoniae ATCC 700603 was taken as positive control and it was kindly provided by the department of microbiology, BIRDEM, Dhaka. Esch. coli, which was sensitive to ceftazidime, ceftriaxone, cefotaxime and aztreonam was used as negative control.

\section{Screening Test ${ }^{7}$ :}

Standard inoculum of bacterial suspension matched to 0.5 McFarland was made and Muller Hinton agar (MHA) plate was inoculated properly with bacterial suspension. Ceftazidime $(30 \mu \mathrm{g})$, Ceftriaxone $(30 \mu \mathrm{g})$, Cefotaxime $(30 \mu \mathrm{g})$

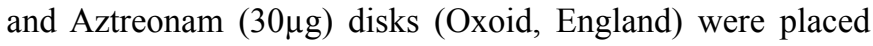
onto MHA plate and incubated overnight at $37^{\circ} \mathrm{C}$ in air. When inhibition zone of any isolate to Ceftazidime $\leq 22 \mathrm{~mm}$ or Aztreonam $\leq 27 \mathrm{~mm}$, or Cefotaxime $\leq 27 \mathrm{~mm}$ or
Ceftriaxone $\leq 25 \mathrm{~mm}$ alone or in combination was found then the isolate was taken as screening test positive ${ }^{8}$.

\section{DDST $^{4,10}$ :}

The MHA plate was inoculated with bacterial suspension matched to 0.5 McFarland. Ceftazidime $(30 \mu \mathrm{g})$, Ceftriaxone $(30 \mu \mathrm{g})$, Cefotaxime $(30 \mu \mathrm{g})$ and Aztreonam $(30 \mu \mathrm{g})$ disks were placed $15 \mathrm{~mm}$ distance centre to centre from amoxiclav disk $(20 \mathrm{mg}$ amoxicillin and $10 \mathrm{mg}$ of clavulanic acid) which was placed at middle. Any extension of inhibition zone of antimicrobial disks(one or more) towards amoxiclav disk confirmed the presence of ESBL.

\section{E test ESBL Method ${ }^{8,10,11}$ :}

Triple ESBL detection (Ezy MICTM from HIMEDIA, India) strip was used. One side of the strip is calibrated with ceftazidime, cefotaxime and cefepimemixture (0.032-4 $\mathrm{mcg} / \mathrm{ml})$ plus fixed concentration of beta lactamase inhibitor mixture (clavulanic acid and tazobactum) having highest concentration tapering downwards. Reverse side carries ceftazidime, cefotaxime and cefepime mixture (0.125-16 $\mathrm{mcg} / \mathrm{ml}$ ) having concentration gradient in opposite direction. Standard bacterial suspension was made and MHA plate was inoculated properly with prepared bacterial suspension. Triple ESBL detection strip was placed on Muller Hinton agar plate. Plates were incubated overnight at $37^{\circ} \mathrm{C}$ in air. The presence of ESBLwas confirmed by the appearance of a phantom zone or when the minimum inhibitory concentration (MIC) of antibiotic mixture side was reduced by $\geq 8$ times in the presence of beta lactamases inhibitor.

\section{Results:}

A total of 124 Gram negative bacteria were subjected to ESBL detection methods. E test was taken as standard for ESBL detection. Screening test detected $69.35 \%(n=86)$ ESBL producing strain, DDST identified $37.1 \%(\mathrm{n}=46) \mathrm{ESBL}$ positive strain and E test ESBL method determined 55.65\% $(n=55)$ ESBL producing isolates.

Table I: Detection of ESBL producing strain by different methods $(n=124)$

\begin{tabular}{lcc}
\hline Name of the test & $\begin{array}{c}\text { \% of ESBL strain } \\
\text { (Positive number) }\end{array}$ & $\begin{array}{c}\text { \% of non ESBL strain } \\
\text { (Negative number) }\end{array}$ \\
\hline Screening test & $69.35(86)$ & $30.65(38)$ \\
DDST & $37.1(46)$ & $62.9(78)$ \\
E test & $55.65(69)$ & $44.35(55)$ \\
\hline
\end{tabular}

$\mathrm{p}$ value $<0.001$ 
Table II: The sensitivity and specificity of screening test against $\mathrm{E}$ test

\begin{tabular}{lccccc}
\hline Screening test & \multicolumn{2}{c}{ E test ESBL method } & Sensitivity & Specificity \\
& Positive & Negative & Total & & \\
\hline Positive & 67 & 19 & 86 & $97.10 \%$ & $65.45 \%$ \\
Negative & 02 & 36 & 38 & & \\
Total & 69 & 55 & 124 & & \\
\hline
\end{tabular}

Table III: The sensitivity and specificity of DDST against $\mathrm{E}$ test

\begin{tabular}{lccccc}
\hline DDST & \multicolumn{2}{c}{$\begin{array}{c}\text { E test ESBL method } \\
\text { Positive }\end{array}$} & Total & Sensitivity & Specificity \\
& 43 & 03 & 46 & $62.31 \%$ & $94.55 \%$ \\
Positive & 26 & 52 & 78 & & \\
Negative & 69 & 55 & 124 & & \\
Total & & & & & \\
\hline
\end{tabular}

\section{Discussion:}

ESBL producing bacteria are resistant to nearly all beta lactam antimicrobialswhich comprise more than 50\% antibiotic uses for systemic infections. Thus ESBL producing Gram negative bacteria make a threat to current beta lactam therapy ${ }^{13}$.

In this study, ESBL positive strains had been identified by screening test, DDST and E test ESBL method. E test is reliable and was taken as standard. Other methods were compare with $\mathrm{E}$ test ${ }^{11}$. Screening test showed many false positive results and exhibited only $65.45 \%$ specificity. Therefore this method could not be suggested to isolate ESBL producing strains ${ }^{11}$.

DDST is a recommended phenotypically confirmatory test but it has many limitations 9 . Evaluation of the double-disk diffusion test against genotypic methods revealed its sensitivity ranging from $79 \%$ to $97 \%$ and specificity ranging from $94 \%$ to $100 \%$. Most important advantage of DDST is that it is technically simple and the interpretation of the test is subjective. However, the sensitivity may be reduced when ESBL activityis very low. ${ }^{2}$ In this study the sensitivity of DDST was $62.31 \%$, making it clear that it missed a fair number of ESBL positive strains. Hence DDST should not be a recommended method for ESBL positive strain isolation in diagnostic laboratory.

The commercially available ESBL Etest strip is a quantitative technique and is widely regarded as the 'gold standard' forESBL detection in clinical laboratories ${ }^{14}$. E test method of this study identified $55.65 \%$ ESBL positive strainamong 124 Gram negative isolates. E test strip carrying combination of cephalosporins has increased its sensitivity. E test technique is an expensive method and this combination makes the technique more expensive. However, it is a recommended method. Clinical laboratorycan use it for confirmation of screening positive isolates rather than as a routine test $\mathrm{t}^{15}$. In addition, early detection of ESBL positive strains can markedly reduce expenditure related to patient management ${ }^{3}$.

Mobile units of Gram-negative bacteria often carry multiple genes for multiple beta-lactamase enzymes such as ESBLs, AmpC beta-lactamases, metallo beta-lactamases and KPC carbapenemases. Simultaneous presence of multiple gene encoded multiple beta-lactamase enzyme in plasmids or transposons warrants that these genes could reach any Gram negative bacterium and can endanger public health ${ }^{2}$. In 2009, a study at Sir Salimullah Medical College detected 36\% ESBL positive isolates among all Gram negative pathogens 7 . In 2012, the percentage of ESBL producing isolates reached to $41.3 \%{ }^{8}$. Current study detected $55.65 \%$ ESBL positive strain from this same institution in 2013. If this upward trend continuesthe acquisition of resistance would go beyond control. To prevent this upward trend of resistant pathogens, routine identification of ESBL producing strains is mandatory by a reliable cost effective phenotypic method from each clinical specimen.

The phenotypic ESBL isolation methods lose their sensitivity if the isolate produces an additional AmpC or metallo betalactamases which are not inhibited by clavulanic acid and tazobactam. This can be a significant clinical problem. Therefore, phenotypic detection should be accompanied by genotypic identification of ESBL genes where facilities are available ${ }^{3}$.

\section{Conclusion:}

The ESBL producing strains are increasing day by day at an alarming rate. These resistant pathogens are one of the important causes of uncontrolled infection. To reduce the dissemination of resistant pathogens specific treatment is urgently needed. As ESBL producing strains are resistant to almost all beta lactam antibiotics except carbapenem early detection is essential. Thereby in clinical laboratory, a reliable cost effective phenotypic method should be applied to confirm screening positive ESBL isolates. 


\section{Reference:}

1. Bush K, Jacoby GA, Medeiros AA: A functional classification scheme for $\beta$-lactamases and its correlation with molecular structure. Antimicrob Agents Chemother 1995; 39:1211-1233.

2. Bush K. Bench-to-bedside review: The role of $\beta$ lactamases in antibiotic-resistant Gram-negative infections. Bush Critical Care 2010;14: 224.

3. Paterson DL. and Robert A. Bonomo.Extended-Spectrum $\beta$-Lactamases: a Clinical Update Clinical Microbiology Reviews 2005;18 (4): 657-686.

4. Rahman NMW. Detection of CTX-M gene in extended spectrum $\beta$-lactamase (ESBL) producing Escherichia coli and Klebsiella species of different hospitals [M.Phil Thesis] Dhaka: Department of Microbiology, Sir Salimullah Medical College, The University of Dhaka 2009:p. 114.

5. Saha M. Phenotypic detection of extended spectrum betalactamase(ESBL) producing isolates and molecular detection of SHV gene by PCR [M.Phil Thesis] Dhaka:Department of Microbiology, Sir Salimullah Medical College, The University of Dhaka 2012:p.109.

6. Tsering DC, DasS and Singh TSK. Extended Spectrum Beta-lactamase Detection in Gram-negative Bacilli of Nosocomial Origin. Journal of Global Infectious Diseases 2009;1(2):87-92.

7. Bush and Jacoby GA. Updated Functional Classification of $\beta$ - Lactamases. Antimicrobial Agents of Chemotherapy 2010; 54(3):969.

8. Clinical and Laboratory Standard Institute M100-S23, Performance Standards for Antimicrobial Susceptibility Testing; Twenty-Third Informational Supplement: Clinical and Laboratory standard Institute:2013.
9. European Society of Clinical Microbiology and Infectious diseases,EUCAST guidelines for detection of resistance mechanisms and specific resistances of clinical and/or epidemiological importance:EUCAST;2012.

10. Chatterjee SS, Karmacharya R, Madhup SK, Gautam V, Das A, Ray P. High prevalence of co-expression of newer $\beta$-lactamases (ESBLs, Amp-C- $\beta$-lactamases, and metallo$\beta$-lactamases) in gram-negative bacilli. Indian $\mathrm{J}$ Med Microbiol 2010;28:267-8

11. Rishi DHP and JohnC. ESBLs: A Clear and Present Danger. Hindawi Publishing Corporation, Critical Care Research and Practice Volume 2012, Article ID 625170, 11 pages.

12. Johann DDP, LauplandKB. Extended-spectrum $\beta$ lactamase-producing Enterobacteriaceae: an emerging public-health concern. Available at http://infection.thelancet.com Vol 8 March 2008.

13. Krishnamurthy V, Vijaykumar GS. and Nagaraj ER. Phenotypic and Genotypic Methods for Detection of Extended Spectrum $\beta$ Lactamase Producing Escherichia coli and Klebsiellapneumoniae Isolated from Ventilator Associated Pneumonia. Journal of Clinical and Diagnostic Research 2013;7(9):1975-1978.

14. M'Zali FH, Chanawong A, Kerr KG, Birkenhead D andHawkey PM. (2000). Detection of extended-spectrum $\beta$-lactamases in members of the family Enterobacteriaceae: comparison of the MAST DD test, the double disc and the Etest ESBL. J AntimicrobChemother 2000;45: 881-885.

15. Dashti AA, West P, Paton R, and Amyes SGB. Characterization of extended-spectrum $\beta$-lactamase (ESBL)- producing Kuwait and UK strains identified by the Viteck system, and subsequent comparison of the Viteck system with other commercial ESBL-testing systems using these strains; Journal of Medical Microbiology 2006;55:417-421. 